\title{
STRONG COMMUTATIVITY PRESERVING GENERALIZED DERIVATIONS ON TRIANGULAR RINGS
}

\author{
He Yuan, Yao Wang, Yu Wang And YiQiu Du
}

\begin{abstract}
Let $\mathscr{U}=\operatorname{Tri}(A, M, B)$ be a triangular ring such that either $A$ or $B$ has no nonzero central ideals. It is shown that every pair of strong commutativity preserving generalized derivations $g_{1}, g_{2}$ of $\mathscr{U}$ (i.e., $\left[g_{1}(x), g_{2}(y)\right]=[x, y]$ for all $x, y \in \mathscr{U}$ ) is of the form $g_{1}(x)=\lambda^{-1} x+[x, u]$ and $g_{2}(x)=\lambda^{2} g_{1}(x)$, where $\lambda \in Z(\mathscr{U})$, the center of $\mathscr{U}$, and $u \in \mathscr{U}$ with $u[\mathscr{U}, \mathscr{U}]=0=[\mathscr{U}, \mathscr{U}] u$. As consequences, every pair of strong commutativity preserving generalized derivations on upper triangular matrix rings and nest algebras is determined.
\end{abstract}

Mathematics subject classification (2010): 15A78, 47L35, 16U80, 16W25.

Keywords and phrases: Triangular ring, upper triangular matrix ring, nest algebra, strong commutativity preserving maps, generalized derivations.

\section{REFERENCES}

[1] E. Alba, N. ARgac, De V. Filippis, Generalized derivations with Engel conditions on one-sided ideals, Comm. Algebra 36 (2008), 2063-2071.

[2] H. E. BeLL, M. N. DAIF, On commutativity and strong commutativity preserving maps, Canad. Math. Bull. 37 (1994), 443-447.

[3] D. Benkovič, D. EREMita, Commuting traces and commmutativity preserving maps on triangular algebras, J. Algebra 280 (2004), 797-824.

[4] N. Boudi, S. Ouchrif, On generalized derivations in Banach algebras, Studia Math. 194 (2009), 81-89.

[5] M. BREŠAR, On the distance of the composition of two derivations to the generalized derivations, Glasgow Math. J. 33 (1991), 89-93.

[6] M. BREŠAR, C. R. Miers, Strong commutativity preserving maps of semiprime rings, Canad. Math. Bull. 37 (1994), 457-460.

[7] W.-S. Cheung, Commuting maps of triangular algebras, J. London Math. Soc. 63 (2001), 117-127.

[8] W.-S. Cheung, Mappings on triangular algebras, PhD Dissertation, University of Victoria, 2000.

[9] K. R. Davidson, Nest Algebras, in: Pitman Res. Notes Math. Ser., vol. 191, Longmans, Harlow, 1988.

[10] Q. Deng, M. Ashraf, On strong commutativity preserving maps, Results Math. 30 (1996), 259-263.

[11] Y. Q. DU, Y. WANG, k-Commuting maps on triangular algebras, Linear Algebra Appl. 436 (2012), 1367-1375.

[12] D. EREmita, Functional identities of degree 2 in triangular rings, Linear Algebra Appl. 438 (2013), 584-597.

[13] DE V. FILIPPIS, An Engel condition with generalized derivations on multilinear polynomials, Israel J. Math. 162 (2007), 93-108.

[14] B. Hvala, Generalized derivations in rings, Comm. Algebra 26 (1998), 1147-1166.

[15] T.-K. LEE, Generalized derivations of left faithful rings, Comm. Algebra 27 (1999), 4057-4073.

[16] T.-K. LeE, Y. Zhou, An identity with generalized derivations, J. Algebra Appl. 8 (2009) 307-317.

[17] P.-B. LiAO, C.-K. LiU, On generalized Lie derivations of Lie ideals of prime algebras, Linear Algebra Appl. 430 (2009), 1236-1242.

[18] J.-S. Lin, C.-K. LiU, Strong commutativity preserving maps on Lie ideals, Linear Algebra Appl. 428 (2008), 1601-1609. 
[19] J.-S. LIN, C.-K. LIU, Strong commutativity preserving maps in prime rings with involution, Linear Algebra Appl. 432 (2010), 14-23.

[20] C.-K. LIU, Strong commutativity preserving generalized derivations on right ideals, Monatsh Math. 166 (2012), 453-465.

[21] J. MA, X. W. XU, F. W. NIU, Strong commutativity preserving generalized derivations on semiprime rings, Acta Math. Sin. (Engl. Ser) 24 (2008), 1835-1842.

[22] X. F. QI, J. C. HoU, Strong commutativity preserving maps on triangular rings, Operators and Matrices 6 (2012), 147-158.

[23] Y. WANG, Additivity of multiplicative maps on triangular rings, Linear Algebra Appl. 434 (2011), $625-635$. 